\title{
A roar for INCENP
}

In animal cells cytokinesis begins shortly after the separation of sister chromatids, when the proposed interaction of the microfilament and microtubule mitotic spindles leads to formation of the actomyosin contractile ring at the equatorial cortex. Although many genes have been implicated either in cytokinesis, such as inner-centromere protein (INCENP), RhoGTPases and mitotic-like kinesins (MLK), or in cytokinesis and chromosome segregation, such as the Aurora-family kinase Aurora-B/AIR2, the exact mechanism by which these processes are regulated is unknown. Three recent papers, by Adams et al. (Curr. Biol. 10, 1075-1078; 2000), Severson et al. (Curr. Biol. 10, 1162-1171; 2000) and Kaitna et al. (Curr. Biol. 10, 1172-1181; 2000), have shed light on the precise functions of Aurora-related kinases, INCENP and the MLK MKLP1/ZEN-4 in both chromosome segregation and cytokinesis.

Using Xenopus laevis eggs, Adams and colleagues demonstrated that Xenopus INCENP is stockpiled in an exclusive complex with Aurora-B/X-AIRK2 (Xenopus Aurora-related serine-threonine kinase) and that the two proteins co-localize during anaphase (picture shows INCENP in red and Aurora-B/AIRK2 in green). As work using budding yeast has previously shown that Sli5p (an INCENP homologue) and Ipl1p (an Aurora-kinase homologue) can interact, this led the authors to propose that INCENP and Aurora-B/AIRK2 interact with each other, a hypothesis that they confirmed using in vitro expression studies. In human cells, transient expression of dominant negative INCENP prevented correct localization of the endogenous protein during metaphase and led to defects in Aurora-B/X-AIRK2 localization. Could it be that INCENP is required to target Aurora-B/AIRK2 to centromeres and the central spindle?

In an independent study, Kaitna and colleagues identified two Caenorhabditis elegans proteins, ICP-1 and ICP-2, that share homology with vertebrate INCENP, and confirmed the above results. Using RNA interefence (RNAi) in C. elegans embryos, they showed that both ICP-1 and Aurora-B/AIR-2 are essential for chromosome segregation and cytokinesis. They went on to demonstrate that $C$. elegans Aurora-B/AIR-2 and ICP-1 can also interact in vitro, and that their mammalian homologues interact in vivo. The authors also showed that localization of AIR-2 in C. elegans relies on INCENP.

On the basis of these two studies, it seems that INCENP localizes AIR-2 to chromosomes during metaphase, and that it also has a role in cytokinesis that is directly linked to its ability to correctly localize Aurora-B/AIR-2. But how does Aurora-B/AIR-2 drive cytokinesis and chromosome segregation?

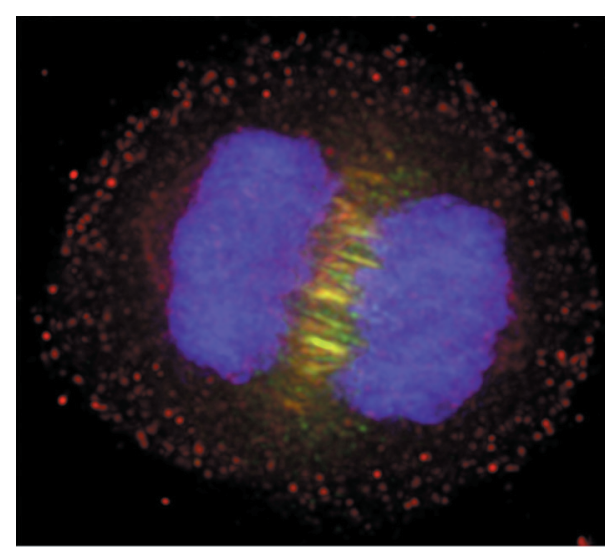

SALLY WHEATLEY AND WILLIAM C. EARNSHAW

In C. elegans embryos with conditional mutations in Aurora-B/AIR-2, Severson and colleagues showed that the MLK, CeMKLP1/ZEN-4, did not localize to the spindle midzone as compared with wild-type embryos, preventing cytokinesis from proceeding. It was previously shown that null mutations in CeMKLP1/ZEN-4 lead to late cytokinetic defects in $C$. elegans embryos but have no effect on DNA segregation. Not unexpectedly, Severson and colleagues observed an in vitro interaction between Aurora-B/AIR-2 and CeMLKP1/ZEN-4.

From all these findings it seems that Aurora-B/AIR-2 does have two separate functions - one in DNA segregation and one in cytokinesis. How Aurora-B/AIR-2 regulates chromosome segregation, along with INCENP, is unknown, but it could be through interactions with the cohesin machinery, which was recently implicated in sister-chromatid separation, or through action on the kinetochores. After chromosome segregation Aurora-B/AIR-2 may interact with CeMLKP1/ZEN-4 to stabilize its localization at the central spindle. As CeMLKP1/ZEN-4 is needed to assemble the central spindle and interacts genetically with CYK-4, a RhoGTPase activating protein, it seems that this could lead to the completion of cell division. These important studies have begun to unravel the role of Aurora-related kinases during cell division, and we await with interest the identification of the precise mechanisms that control their action.

SARAH GREAVES 